\title{
Transcranial Electric Stimulation Entrains Cortical Neuronal Populations in Rats
}

\author{
Simal Ozen, ${ }^{1}$ Anton Sirota, ${ }^{1,2}$ Mariano A. Belluscio, ${ }^{1}$ Costas A. Anastassiou, ${ }^{3,4}$ Eran Stark, ${ }^{1}$ Christof Koch, ${ }^{3,5}$ \\ and György Buzsáki ${ }^{1}$ \\ ${ }^{1}$ Center for Molecular and Behavioral Neuroscience, Rutgers University, Newark, New Jersey 07102, ${ }^{2}$ Centre for Integrative Neuroscience, University of \\ Tübingen, Tübingen 72076, Germany, ${ }^{3}$ Division of Biology, California Institute of Technology, Pasadena, California 91125, ${ }^{4}$ Department of Bioengineering, \\ Imperial College, London, London SW7 2AZ, United Kingdom, and 5Department of Brain and Cognitive Engineering, Korea University, Seoul 136-701, Korea
}

Low intensity electric fields have been suggested to affect the ongoing neuronal activity in vitro and in human studies. However, the physiological mechanism of how weak electrical fields affect and interact with intact brain activity is not well understood. We performed in vivo extracellular and intracellular recordings from the neocortex and hippocampus of anesthetized rats and extracellular recordings in behaving rats. Electric fields were generated by sinusoid patterns at slow frequency $(0.8,1.25 \mathrm{or} 1.7 \mathrm{~Hz})$ via electrodes placed on the surface of the skull or the dura. Transcranial electric stimulation (TES) reliably entrained neurons in widespread cortical areas, including the hippocampus. The percentage of TES phase-locked neurons increased with stimulus intensity and depended on the behavioral state of the animal. TES-induced voltage gradient, as low as $1 \mathrm{mV} / \mathrm{mm}$ at the recording sites, was sufficient to phase-bias neuronal spiking. Intracellular recordings showed that both spiking and subthreshold activity were under the combined influence of TES forced fields and network activity. We suggest that TES in chronic preparations may be used for experimental and therapeutic control of brain activity.

\section{Introduction}

Since neurons are electrically charged structures, extracellular fields generated around them affect their excitability (Bishop and O'leary, 1950; Terzuolo and Bullock, 1956; Rall and Shepherd, 1968; Jefferys, 1981, 1995; Taylor and Dudek, 1982; Traub et al., 1985, 1999; Chan and Nicholson, 1986; Tranchina and Nicholson, 1986; Holt and Koch, 1999; McIntyre and Grill, 1999; Francis et al., 2003; Bédard et al., 2004; Gold et al., 2006; Logothetis et al., 2007). The impact of extracellular fields on neuronal excitability raises three important issues. The first critical question is the extent to which local field potentials, generated by either normal or pathological activity of neuronal assemblies, affect neuronal firing by nonsynaptic mechanisms (Noebels and Prince, 1978; Jefferys and Haas, 1982; Jefferys, 1995; Francis et al., 2003; Parra and Bikson, 2004; Anastassiou et al., 2010). The second issue is whether exposure to electric and magnetic fields in modern environments presents health risks (Adey, 1981; Bawin et al., 1984; Jefferys et al., 2003). The third question is whether externally applied electric or magnetic fields can be exploited for the treatment of neurological and psychiatric diseases (Rosenthal, 1972; Barker et al., 1985; George et al., 1999; Walsh and Cowey, 2000;

Received 0ct. 22, 2009; revised July 8, 2010; accepted July 14, 2010.

This work was supported by National Institutes of Health Grants NS034994 and NSF 0613414, the James M. McDonnell Foundation, the Pew Charitable Trust, the Mathers Foundation, the Gimbel Fund, the National Science Foundation, the World Class University program through the National Research Foundation of Korea, funded by the Ministry of Education, Science and Technology (R31-2008-000-10008-0), and the Engineering and Physical Science Research Council. We thank Thomas Allen, Bart Krekelberg, David A. McCormick, Kenji Mizuseki, Denis Paré, and Thomas Radman for their critical input on the manuscript.

Correspondence should be addressed to György Buzsáki, Center for Molecular and Behavioral Neuroscience, Rutgers University, 197 University Avenue, Newark, NJ 07102. E-mail: buzsaki@axon.rutgers.edu.

D01:10.1523/JNEUROSCI.5252-09.2010

Copyright $\odot 2010$ the authors $\quad 0270-6474 / 10 / 3011476-10 \$ 15.00 / 0$
Gluckman et al., 2001; Francis et al., 2003; Walsh and Pascual-Leone, 2003; Fitzgerald et al., 2006; Fregni et al., 2006; Webster et al., 2006; Radman et al., 2007a; Nitsche et al., 2009).

Transcranial magnetic stimulation (TMS) is a leading experimental tool for studying the human brain. The pulsed magnetic field can temporarily excite or inhibit specific areas and can even produce long-term changes (Barker et al., 1985; Hallett, 2000; Walsh and Cowey, 2000; Fitzgerald et al., 2006). A disadvantage of TMS is that large and heavy coils are required for generating magnetic fields. The large size and apparent visibility of TMS instruments make this method less than ideal for long-term outpatient treatment.

Experimental and clinical applications of transcranial electric stimulation (TES) in humans have been traditionally confined to transcranial direct current stimulation (tDCS) (cf. Nitsche et al., 2009). However, in a recent study on sleep-assisted memory consolidation, Marshall et al. (2006) showed a significant enhancement of memory recall induced by oscillating current TES at 0.75 Hz. In another study (Terney et al., 2008), TMS-evoked responses were enhanced by random noise stimulation applied by TES. Unfortunately, the direct electrophysiological effects of the stimulation could not be studied, due to TES-induced artifacts. The success of those studies emphasizes the need for a thorough physiological understanding of TES-induced effects on neuronal activity in the intact brain. Prompted partially by these human studies, we investigated the neurophysiological effects of TES in anesthetized and chronically implanted rats. We found that applied electric fields entrained spiking activity of neurons in widespread cortical areas. The effect depended on both the intensity and the polarity of TES and was influenced by the ongoing state changes of brain activity. Chronic application of TES, therefore, 
is an effective tool for investigating and modifying neuronal activity in the intact brain.

\section{Materials and Methods}

Animals and surgery. All procedures followed the rules and regulations of the National Institute of Health Guide for the Care and the use of Laboratory Animals and were approved by the Institutional Animal Care and Use Committee at Rutgers. For acute experiments, male Sprague Dawley rats ( $n=16 ; 100-400 \mathrm{~g}$; Hilltop Laboratories) were anesthetized with urethane $(1.25 \mathrm{~g} / \mathrm{kg}$, i.p.) followed by hourly supplements of ketamine/ xylazine mixture (18 and $1.8 \mathrm{mg} / \mathrm{kg}$, i.m.). Body temperature was monitored and kept constant at $37^{\circ} \mathrm{C}$ using a heating pad. Paw-withdrawal reflex was periodically checked. Atropine-sulfate $(0.03 \mathrm{mg} / \mathrm{kg})$ was subcutaneously administered to aid breathing. For neocortical recordings, three different types of high-density silicon probes were used (Olsson et al., 2005). The first probe, used in 5 acute experiments, had 4 shanks spaced at $200 \mu \mathrm{m}$, each with 8 recording sites staggered at $20 \mu \mathrm{m}$ vertical spacing (Acreo). The second probe, used in 5 acute experiments, had 5 shanks spaced at $200 \mu \mathrm{m}$, each with 12 recording sites staggered at $15 \mu \mathrm{m}$ vertical spacing; the middle shank had 4 additional recording sites at 250 $\mu \mathrm{m}$ spacing, above the highest electrode site (Acreo). The probes were placed in the left medial prefrontal cortex; with the tip targeted at anteroposterior (AP) $2.8 \mathrm{~mm}$, mediolateral (ML) $0.5 \mathrm{~mm}$, and $1.5-3.0 \mathrm{~mm}$ from the dura. The third probe used in the remaining acute experiments had 32 recording sites in $50 \mu \mathrm{m}$ vertical spacing on one shank (NeuroNexus) and was placed in the somatosensory area/hippocampus at AP $-3.5 \mathrm{~mm}$ and ML $2.5 \mathrm{~mm}$ from bregma and $2.4-3.5 \mathrm{~mm}$ from the dura. The same single-shank linear probe was used in mapping the effect of TES. Stainless steel screws for reference and ground electrodes were implanted above the cerebellum. In 3 of the experiments, a borosilicate glass micropipette $(30-70 \mathrm{M} \Omega$ ) filled with $2 \%$ Biocytin in $1 \mathrm{M} \mathrm{K}$-acetate, $\mathrm{pH}$ 7.4 , was vertically inserted into somatosensory cortex at AP $-3.3 \mathrm{~mm}$, ML $2.0 \mathrm{~mm}$ from bregma for intracellular recording. Ag-chloridecovered Ag wire was placed in the neck muscle tissue to serve as reference electrode. The recorded cells were loaded with Biocytin through iontophoresis with $0.5-0.8 \mathrm{nA}$ current injections $(0.2-3 \mathrm{~h})$.

For chronic experiments, three male Long-Evans rats (250-350 g from Charles River Laboratories) were implanted with stimulating and recording electrodes. For rat 1 , six single wires $(20 \mu \mathrm{m}$ in diameter) were implanted in the left hemisphere at $2 \mathrm{~mm}$ anteroposterior spacing from $3.2 \mathrm{~mm}$ to -6.8 $\mathrm{mm}$ relative to bregma and $1.5 \mathrm{~mm}$ from the midline in the left hemisphere, and three wires were implanted in the right hemisphere $3.2 \mathrm{~mm}$ to $-0.8 \mathrm{~mm}$ relative to bregma, also $1.5 \mathrm{~mm}$ from the midline. All wires were targeting the deep neocortical layers. In addition, four single wires, attached to a movable microdrive, were implanted above the right hippocampus at AP $-3.5 \mathrm{~mm}$, ML $1.2 \mathrm{~mm}$ from bregma. In rat 2, a 32-site single-shank silicon probe (staggered at $20 \mu \mathrm{m}$ spacing) was implanted in the right hemisphere at AP $-3.7 \mathrm{~mm}$ and ML $1.2 \mathrm{~mm}$ from bregma. In addition, four tetrodes on a movable drive were implanted in the right hemisphere at $\mathrm{AP}-4.9 \mathrm{~mm}, \mathrm{ML}$ $3.0 \mathrm{~mm}$ from bregma. Two additional wire electrodes were also implanted in the right somatosensory cortex. In rat 3, a 4-shank silicon probe (600 $\mu \mathrm{m}$ shank intervals; 16 recording sites with $50 \mu \mathrm{m}$ spacing on each shank; Acreo), attached to a movable drive, was implanted in the right hemisphere parallel with the long axis of the hippocampus with the tips at AP $-3.6 \mathrm{~mm}$, ML $0.5 \mathrm{~mm}$ and AP $-4.5 \mathrm{~mm}$, ML $2.1 \mathrm{~mm}$ from bregma. In addition, four tetrodes, on separately movable drives, were implanted in the left hemisphere. Chronic recordings were performed while the animal was sleeping or resting in its home-cage, except for the behavioral exploration experiments.

For stimulation, a spiral was formed from $200 \mu \mathrm{m}$ stainless steel wire $(\sim 3 \mathrm{~mm}$ in diameter; Fig. $1 A)$. One such electrode was implanted on each temporal bone and on the calvarium midline above the olfactory bulb during surgery (Fig. 1 $A a$ ). The temporal bone electrodes were $\sim 6$ $\mathrm{mm}$ posterior from the midline electrode. The surface facing the muscle and skin was insulated, whereas the surface facing the skull was conducting. In other (acute) experiments, stimulation was provided by a pair of silver wires placed into grooves in the skull or screws driven halfway into the skull bone (Fig. $1 \mathrm{Ab}, \mathrm{c}$ ). In some acute experiments, capton-based epidural grids $(2.5 \mathrm{~mm} \times 4 \mathrm{~mm})$, placed between the bone and dura were used (Fig. $1 \mathrm{Ad}$ ). Constant current stimulation was used in 6 acute exper- iments, whereas constant voltage stimulation was used for the remaining experiments ( $n=10$ for acute and $n=17$ for chronic experiments).

At the end of the experiments, the animals were deeply anesthetized and transcardially perfused with $\sim 50 \mathrm{ml}$ of cold saline $(0.9 \%)$ followed by $\sim 400 \mathrm{ml}$ of buffered fixative ( $4 \%$ paraformaldehyde with glutaraldehyde $0.5 \%$ in $0.1 \mathrm{~m}$ PBS, pH 7.4). Brains were postfixed at $4^{\circ} \mathrm{C}$ overnight, and then kept in $0.1 \mathrm{M}$ PBS. Brains were sliced into coronal sections $(50-100 \mu \mathrm{m})$. For the single-cell verification, the Biocytin was processed by the Avidin-Biotin-HRP complex method (Vector Labs) and the color was revealed by a chromagen (DAB-Ni). The layer organization was revealed by NeuN or Nissl staining of the cell bodies.

Recording, stimulation and data processing. Extracellular signals were amplified $(1000 \times)$ and wide-bandpass filtered $(1 \mathrm{~Hz}-5 \mathrm{kHz})$. Intracellular signals were buffered $(1 \times)$ and amplified by a DC amplifier (Axoprobe 1A; Molecular Devices). Signals were continuously acquired at 20 $\mathrm{kHz}$ on one or two synchronized 64 channel DataMax systems (16 bit resolution; RC Electronics). All analyses were conducted off-line. The stimulation signal was directly fed from the stimulator output (STG1008 Stimulus Generator; Multi Channel Systems) to one of the recording channels. The peak-to-peak amplitude of the stimulator output is referred to as the stimulation intensity. All intensity values are given as peak-to-peak amplitudes. For LFP display and analysis, the volumeconducted stimulus (TES) artifact was removed as follows. The TESrelated component of the LFP was estimated using least-square fit between the stimulation signal and the LFP. Time delay parameter and the scaling factor of the fit were adjusted dynamically in $10 \mathrm{~s}$ windows, sliding in $7 \mathrm{~s}$ steps. By subtracting the best-fit component from the LFP a continuous artifact-free signal was constructed (supplemental Fig. 4, available at www.jneurosci.org as supplemental material). Spectrograms of the LFP trace (see Fig. $6 \mathrm{~A}$ ) were computed using windows of $4 \mathrm{~s}$ lengths, sliding in $1 \mathrm{~s}$ steps.

Unit activity was detected from the high-pass filtered $(>0.8 \mathrm{kHz})$ trace and single units were semiautomatically isolated using Klusta-Kwik (Harris et al., 2000), followed by a manual refinement using a custommade software (Hazan et al., 2006), which used spike waveforms, autocorrelograms and cross-correlograms. To ensure that only high quality units were used in the analyses, we set two criteria for spike inclusion. First, the amplitude of the spike should be $>60 \mu \mathrm{V}$. This value is based on our previous observations that smaller amplitude spikes result in clusters bordered closely by other clusters (at least in the hippocampal CA1 region) (Henze et al., 2000). Second, the peak-to-peak spike amplitude (p2p) should exceed 3.6 times the temporally local background noise, determined according to the $\mathrm{SD}$ of the signal in the immediate vicinity of each spike (between $0.8-0.4 \mathrm{~ms}$ before and $0.4-0.8 \mathrm{~ms}$ after the extracellular trough), and scaled by the natural logarithm of the number of samples across which the SD was computed. This definition of spike signal/noise, $\mathrm{SNR}=\mathrm{p} 2 \mathrm{p} / \mathrm{SD}$ (noise) $/ \ln (n)$, is independent of the number of samples used to compute the noise and is asymptotically 1 for bandpass-filtered white noise (supplemental Fig. 1, available at www. jneurosci.org as supplemental material). Although these strict criteria likely eliminated several true spikes, it reduced the likelihood of obtaining false-positive results. A cluster was classified as "multiunit" if the autocorrelogram lacked a clear refractory period. Multiunit clusters recorded from the same electrode (e.g., the shank of the probe) were merged into a single multiunit cluster to avoid potential oversampling by neighboring recording sites. Short-latency $(<5 \mathrm{~ms})$ temporal interactions with other isolated neurons were used to identify single neurons as putative excitatory or inhibitory cells (Bartho et al., 2004; Isomura et al., 2006; Fujisawa et al., 2008; Sirota et al., 2008). The significance of monosynaptic connections was evaluated according to the global and point-wise significance values obtained by jittering $(n=500)$ the cross-correlograms between single-unit pairs. Cross-correlograms with significant peaks $<5 \mathrm{~ms}$ of the reference neuron spikes were regarded as monosynaptically connected (Fujisawa et al., 2008).

TES stimulation was applied multiple times (minimum of 5 trials) for a given stimulation protocol, that varied in intensity, frequency $(0.8-1.7$ $\mathrm{Hz}$ ) and duration ( 15 cycles to 60 cycles; 1 min long for few acute experiments) across sessions. Each stimulation trial was followed by a stimulation-free period ( $>40 \mathrm{~s}$, except for short-duration stimulation 
trials, for which it was $10 \mathrm{~s}$ ). The signal was a sinusoid waveform. In most sessions, stimulation was applied through 3 stimulating electrodes (3-poles), such that the same polarity was applied to both hemispheres (side) versus the opposite polarity applied to the center (frontal) electrode (Fig. 1Aa,d). This configuration yielded synchronous electric fields in both hemispheres. In a few experiments, bipolar stimulation was used (Fig. $1 A b, c$ ). Trials using the same stimulation protocol were combined for statistical analysis.

To assess the effect of stimulation on unit firing, each spike was assigned to the instantaneous phase of the reference TES signal obtained by Hilbert transformation. The significance of phasemodulation was evaluated using Kuiper's test of random deviation from uniformity on circular data (Fisher, 1993). This omnibus test was preferred over the more standard Rayleigh test, since our findings (such as bimodal phase preference) precluded having a predefined assumption regarding the modality of the TES-induced phase entrainment and thus the application of conventional (unimodal) phase entrainment measures (e.g., Rayleigh statistic). Since omnibus tests require a sufficiently large sample of data, we applied 2 criteria a) a minimum number of spikes (at least 250 per stimulation protocol) and b) a minimum number of trials (at least 5 ) for a given stimulation intensity, frequency and configuration. Because both multiunits and single units had different number of spikes and both were tested against the same uniformity hypothesis, we examined whether the number of spikes biased our results. A fixed number of spikes $(n=600$, $n=1000$ ) was randomly chosen from a given TES session and tested against uniformity. Such subsampling did not show any particular trend substantially different from the case when all spikes were included. Probabilities $<0.01$ were regarded as significant.

For the color-coded population display, the spike count of each unit was normalized by subtracting the mean spike count of that unit from the spike count of each bin and dividing by the SD ( $20^{\circ}$ phase bins across one period of TES signal).

To examine the relationship between spikes and the intrinsic slow oscillation, the peak spectral power of LFP (peakF) within the $0.4-10 \mathrm{~Hz}$ frequency range was computed in $3.2 \mathrm{~s}$ windows. The original LFP signal was bandpass filtered around peakF, between $[0.75 \times$ peakF and $1.25 \times$ peakF] Hz, using second order, Butterworth Filter (a built-in Matlab function). The phase of the bandpass filtered LFP was then derived by Hilbert transformation. Phase-modulation of units by the slow oscillation was evaluated by Rayleigh circular statistics, since the unimodal phase modulation by the intrinsic slow oscillation could be safely assumed from previous experimental evidence (Steriade et al., 1993). For the display of cycle-by-cycle unit firing, peri-event time histograms of spike times around the first/last troughs of the reference TES signal were constructed.

For the analysis of the intracellular signal $(<1250 \mathrm{~Hz}$, low-pass), the signal was bandpassed filtered $(0.5-10 \mathrm{~Hz})$ to remove the action potentials. The relationship between the
A
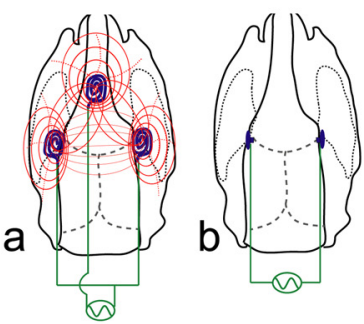

B

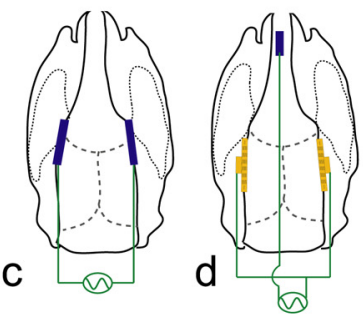

㞻
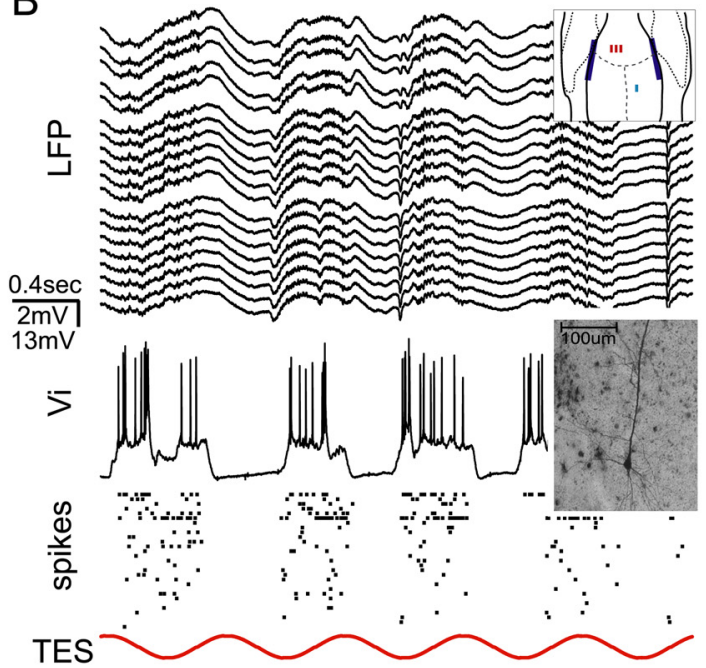

C
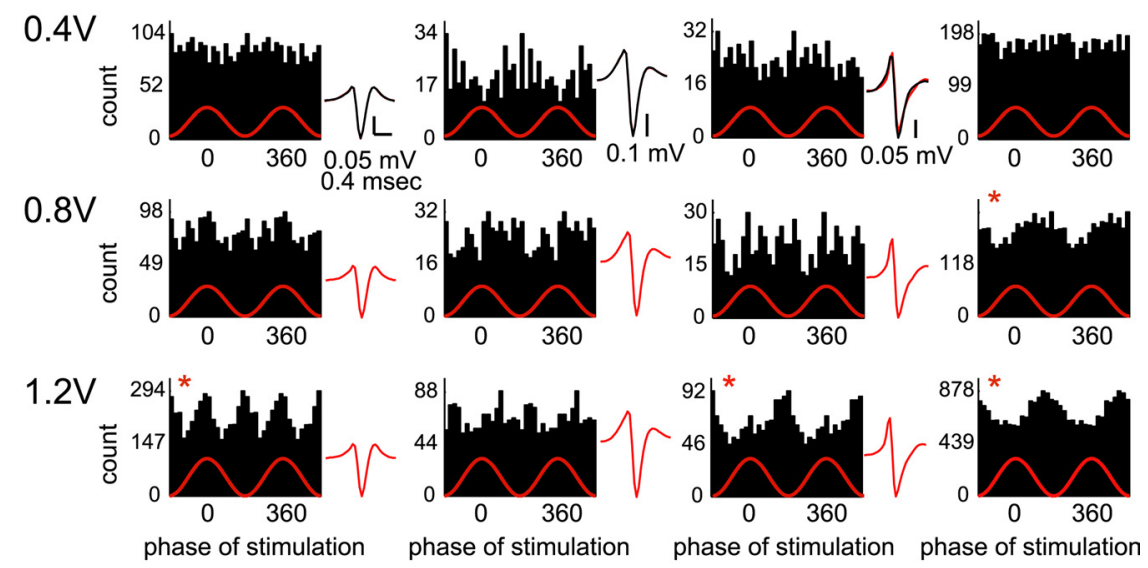

D

\section{$0.4 \mathrm{~V}$}

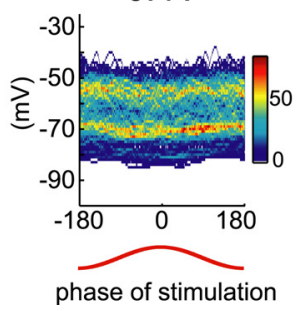

$0.8 \mathrm{~V}$

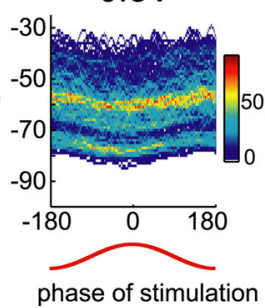

$1.2 \mathrm{~V}$

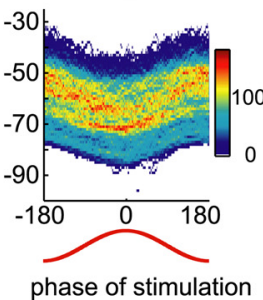

corrected 1.2V -30 .

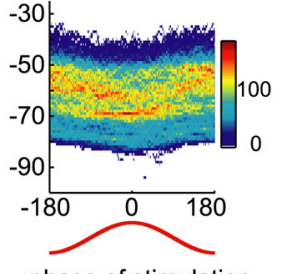

Figure 1. Entrainment of subthreshold and suprathreshold neuronal activity by TES.A, Schematic of 4 electrode configurations used for electric field stimulation. $\boldsymbol{A a}$, Wire coils placed on the skull for chronic stimulation. Red dashed lines, idealized electric fields around the electrodes. The coils on the left and right sides of the skull had the same virtual polarity against the third electrode placed above the frontal cortex. $\boldsymbol{A} \boldsymbol{b}$, Screws driven halfway through the skull. $\boldsymbol{A}$, Wires placed on the skull. $\boldsymbol{A d}$, Flexible cables placed epidurally and a silver wire placed in the oral cavity of the animal. $\boldsymbol{B}$, Multiple-site recording of unit and LFP with a 4-shank silicon probe from the prefrontal cortex (activity from 3 shanks is shown; faulty sites are excluded) and simultaneous intracellular recording from a layer V pyramidal cell in the somatosensory area in an anesthetized rat. Top inset, Locations of the stimulation electrodes, intracellular electrode, green, and the silicon probe, red. Bottom inset, Photograph of the biocytin-labeled neuron. Dots, spikes of extracellularly recorded putative single neurons. Red sinusoid line, applied field. Bipolar stimulation (Ac) at $1.25 \mathrm{~Hz}, 0.4 \mathrm{~V}$ intensity. C, TES phase-locked discharge of 3 example units and 1 intracellularly recorded unit (last column) at 3 different stimulation intensities. Averages of $>300$ stimulation cycles. Average filtered unit waveforms, recorded from the probe site with the largest amplitude unit, are shown for different intensity TES epochs (red). Waveforms during prestimulation epochs (black) are shown in the top row, superimposed on the waveforms recorded during 0.4 VTES. Same timescale applies for all waveforms; amplitude scale is shown for each unit at the top row. Unit on the right is the intracellularly recorded neuron. Red stars indicate significantly phase-modulated units ( $p<0.01$, Kuiper's test). $\boldsymbol{D}$, Illustration of intrinsic network- and extrinsic field-control of neural activity. Joint probability density counts of $V_{i}$ of the layer $V$ neuron shown in $B$ and the phase of the TES signal are shown for different intensities of stimulation; weak $0.4 \mathrm{~V}$, moderate $0.8 \mathrm{~V}$ and strong $1.2 \mathrm{~V}$ applied fields. Stimulation phase is illustrated by the red line. Warmer colors indicate high probability of occurrence. Rightmost panel (corrected $1.2 \mathrm{~V}$ ), $V_{\mathrm{i}}$ corrected by subtracting the signal, extracellularly recorded after withdrawing the pipette from the neuron. Note the coexistence of network-induced $V_{i}$ bimodality (hot color bands at two $V_{i}$ levels) and increasing phase-locking of $V_{i}$ to the applied field as its strength increases (note the increasing differences between $0^{\circ}$ and $180^{\circ}$ of TES of DOWN state probabilities). 
A

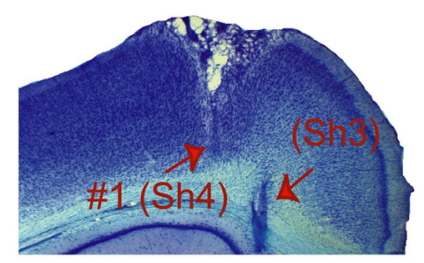

$\mathrm{B}$

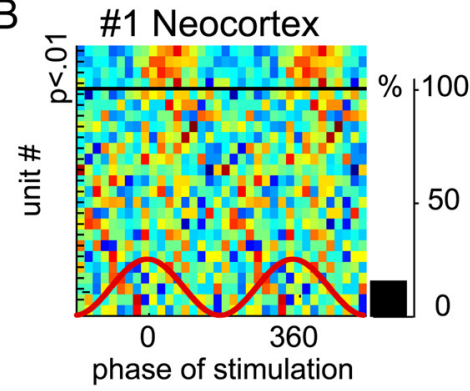

C

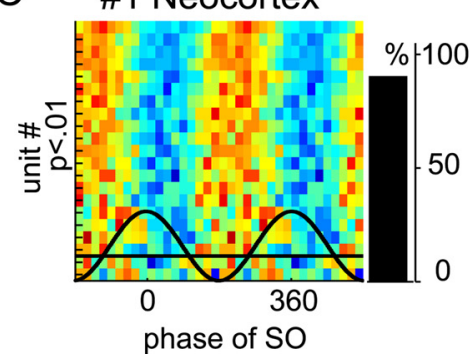

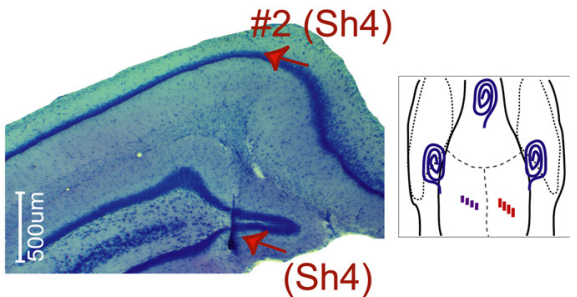

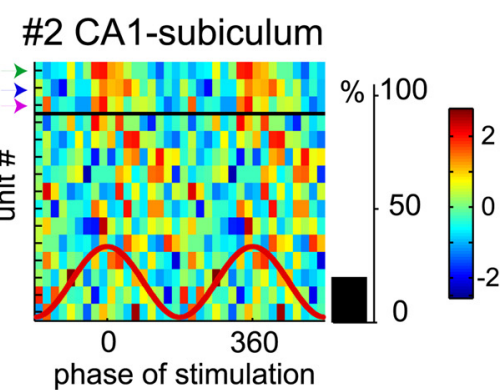

\#2 CA1-subiculum

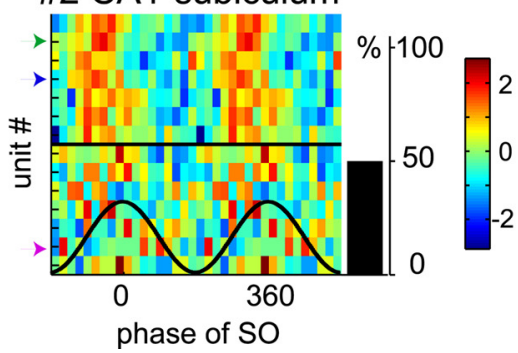

Figure 2. TES entrains neurons in multiple cortical areas. $\boldsymbol{A}$, Histologically verified tracks of a 4-shank silicon probe. Tracks of the two most lateral probe shanks are shown in the neocortex (left, Sh4 and Sh3) and the deep recording sites in the hippocampus (right, sh4). The silicon probe was moved deeper after each recording session. Probe recordings were made at 2 different recording depths (arrows): in the deep layers of the neocortex (1) and hippocampal CA1-subiculum area (2). Right inset, Locations of the stimulation electrodes, silicon probe (red) and tetrodes (purple). $\boldsymbol{B}$, TES entrainment of neurons in 2 recording sessions from the same rat. Each row corresponds to a single-unit or a multiunit cluster, color-coded for standardized firing rate (mean subtracted from instantaneous rate and divided by the SD). Units are sorted for each electrode separately by their $p$-values (increasing from top to bottom) to illustrate the effectiveness of TES-induced unit entrainment. Black lines separate the significantly modulated units $(p<0.01$, above) from the nonmodulated ones below. Side bars, percentage of neurons significantly modulated by TES ( $p<$ 0.01 ; Kuiper's test). Note that TES entrainment of both neocortical and hippocampal neurons occur at the same phase. TES, $1.25 \mathrm{~Hz}$, 3-pole configuration (Fig. 1 $\mathrm{Aa}$ ) across multiple repetitions of at least $7 \mathrm{~min}$ epochs. The fraction of TES-entrained neurons is overestimated because neurons firing at a low rate are not detected properly by clustering algorithms. Left to right, $4.2 \mathrm{~V}, 4.8 \mathrm{~V}$ intensity, respectively, Colors are z-scored (scale on the right). C, Network-controlled phase-locking of neuronal activity to slow oscillations during natural sleep (illustrated by black line) in the absence of TES. SO, slow oscillation. Unit identity is the same as in B. Note strong phase-locking of deep layer neocortical neurons to slow oscillation (Steriade et al., 1993), moderate entrainment of CA1-subicular cells (Isomura et al., 2006). Reference LFP site in each experiment was the deep layer of the contralateral neocortex.

filtered intracellular potential $\left(V_{\mathrm{i}}\right)$ and the phase of the slow oscillation or TES was assessed using joint probability density. This measure allows the assessment of the correlation between network or TES effects and $V_{\mathrm{i}}$ across $\left(20^{\circ}\right)$ phase bins (Isomura et al., 2006). To compensate for the added effect of the TES-induced field on $V_{\mathrm{i}}$, TES was repeated after the pipette was withdrawn from the neuron. The obtained extracellular average polarization for each phase bin of TES signal was subtracted from the $V_{\mathrm{i}}$ to arrive at a field-corrected joint density (Fig. $1 D$ ).

To evaluate the state dependence of stimulation, the $\chi^{2}$ test was performed, comparing the distribution of $p$-values, the significance of phase modulation (Kuiper's test), of each unit. Either two or three $p$-value categories were constructed: not-significant (nS, $p>0.05)$, significant $(\mathrm{S}$, $p<0.005<p \leq 0.05$ ), and highly significant (hS, $p \leq 0.005)$ and the frequency distributions of these categories across conditions (e.g., behavioral state) were evaluated using the $\chi^{2}$ statistics.

\section{Results}

The goal of our experiments was to entrain cortical neurons by exogenously applied electric fields and to determine the underly- ing mechanisms. While generating spatially homogenous fields throughout the entire brain is difficult, generating nonfocal and nearly homogenous fields is possible by large electrodes because the various brain tissues between the electrodes can disperse the charges (Koch, 1999). The stimulation pattern $(n=69$ stimulation sessions in acute, and $n=77$ sessions in chronic experiments) was a $0.8-1.7 \mathrm{~Hz}$ sinusoid, mimicking the frequency of cortical slow oscillations, a common oscillatory rhythm observed in humans and other animals during natural sleep as well as under anesthesia (Steriade et al., 1993; Sirota et al., 2003; Marshall et al., 2006). Using single- or multiple-shank silicon probes and tetrodes (see Materials and Methods) (Sirota et al., 2008), multiple and single units were recorded from several cortical areas ( $n=348$ units in neocortex, $n=$ 36 units in hippocampus). In the experiments under anesthesia, various electrode configurations were used (Fig. 1A). In all acute experiments $(n=3$ animals with epidural flex cable electrodes; $n=5$ animals with screw electrodes and $n=3$ animals with wire electrodes placed on the skull) neurons distant from the stimulation electrodes were driven by TES, the fraction depending on the stimulation intensity (Fig. 1C; supplemental Fig. 2, available at www. jneurosci.org as supplemental material). In three experiments under anesthesia, intracellular recordings were made from layer $\mathrm{V}$ pyramidal neurons in the somatosensory area.

Figure $1 B$ illustrates a short segment of local field potentials (LFP) and unit firing in response to TES. Under anesthesia, the spontaneous slow oscillation, in the frequency range of $1-1.5 \mathrm{~Hz}$, exerted a powerful effect on both the membrane potential and the discharge probability of most neurons $(100 \%$ entrainment of $n=$ 81 neocortical units in prefrontal cortex and somatosensory cortex; $n=5$ animals; Fig. $1 B, D$ ) (Steriade et al., 1993; Isomura et al., 2006). Against this strong network effect, weak TES stimulation $(0.4 \mathrm{~V})$ did not have a profound effect on the neuronal population (only $6 \%$ of units were entrained, $n=16$ units; Fig. 1C; supplemental Fig. $2 B$, available at www.jneurosci. org as supplemental material). At higher intensities $(0.8-1.2 \mathrm{~V})$, a larger fraction of the neurons ( $15 \%$ and $69 \% ; n=13$ and $n=26$ units; respectively) was significantly $(p<0.01)$ phase-locked to the forced TES field and the strength of their entrainment increased as stimulation intensity increased (Fig. $1 C$; supplemental Fig. 2A, available at www.jneurosci.org as supplemental material). The combined effects of synaptically driven network influences and the TES-induced field are illustrated by the distribution of the $V_{\mathrm{i}}$ values of the recorded neuron (Fig. $1 D$ ). The spontaneous, network-induced slow oscillation was coupled with a strong bimodality (UP and DOWN states) (Steriade et al., 1993; Sanchez-Vives and McCormick, 2000) of $V_{\mathrm{i}}$ in the intracellularly 
A

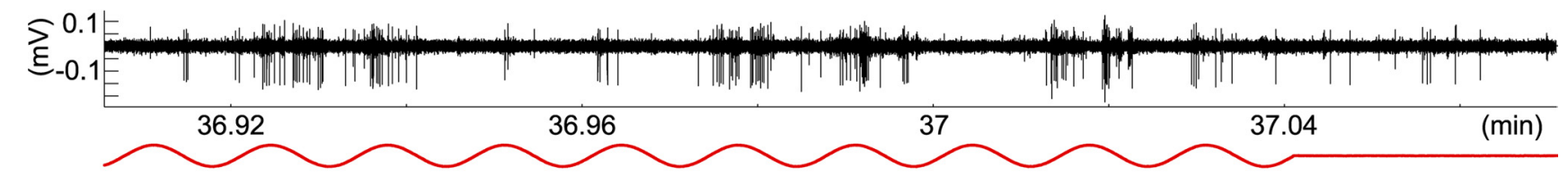

B

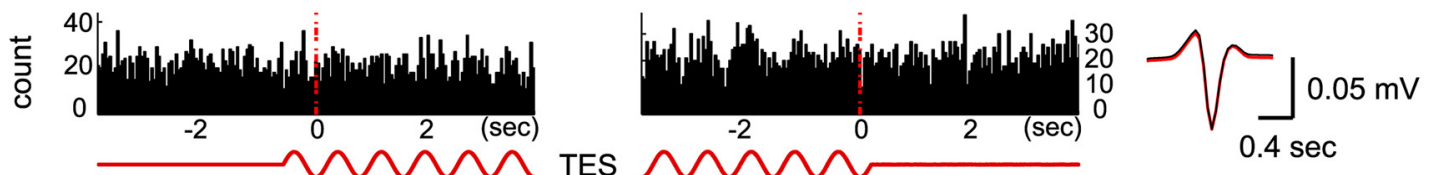

C
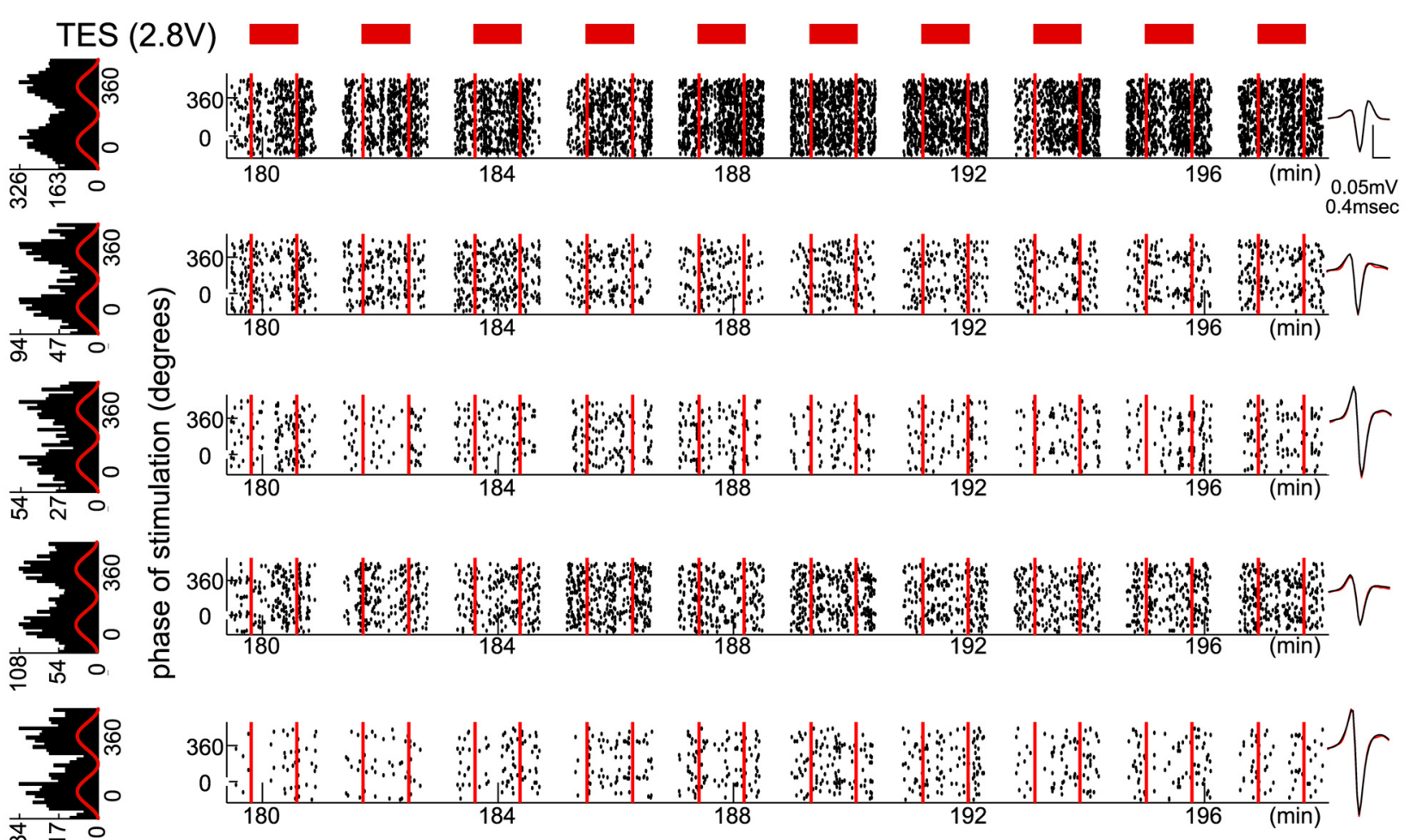

,
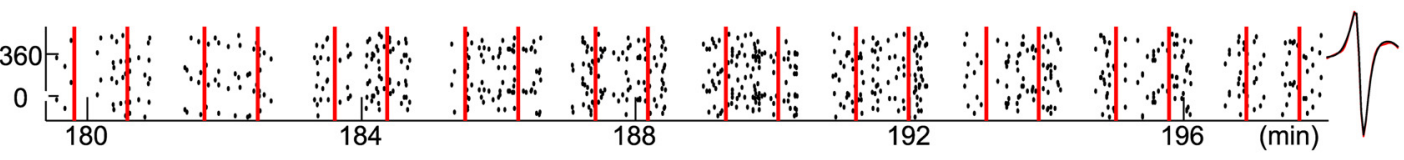

Figure 3. Entrainment of cortical unit activity by TES. $\boldsymbol{A}$, Example filtered (1-5 kHz) extra-cellular trace during TES (1.25 Hz, $4.8 \mathrm{~V}$ intensity). $\boldsymbol{B}$, Averaged TES phase onset and offset-triggered histogram of an example unit (1.25 Hz, 4.8 V intensity, 3-pole configuration as in Fig. $1 \mathrm{Aa})$. Note the lack of persistence of rhythmic entrainment after TES offset (right histogram). Red dotted lines, Troughs of the first and the last cycles. Insets, Average filtered unit waveform recorded during prestimulation epochs (black) and during TES (red). C, Stability of unit entrainment by TES. Five example cortical units recorded simultaneously. Each dot represents a spike, as a function of stimulus phase (ordinate) during 10 successive (1.25 Hz, 2.8 V intensity, 3-pole configuration), 1-min-long stimulation trials (red bars) with 1-min-long resting (stimulation-free) intervals in between. Spikes for $20 \mathrm{~s}$ of presham and postsham periods are shown. Spikes during the remaining stimulationfree periods are not shown to aid the clarity of the display. Note stability of TES entrainment over multiple stimulations. Left, TES phase histograms for the entire session. Insets (right), Average filtered unit waveforms recorded during prestimulation epochs (black) and during TES (red, superimposed).

recorded neurons. During stimulation, the slow oscillationrelated $V_{\mathrm{i}}$ bimodality was preserved but the probability of the UP and DOWN state of the membrane was biased by TES in an intensity-dependent manner (note differences in DOWN state probability between $0^{\circ}$ and $180^{\circ}$ of TES in Fig. $1 D$ ), indicating a combined effect of the network-activated and TES-induced forces. Similar observations were made in two other intracellularly recorded cells (supplemental Fig. 3, available at www. jneurosci.org as supplemental material). To estimate the TESinduced true intracellular potential, TES-stimulation was repeated after the pipette was withdrawn from the cell, and the phase modulation of $V_{\mathrm{i}}$ signal was corrected by subtracting the mean of the TES-induced extracellular potential from $V_{\mathrm{i}}$ at each phase bin $\left(20^{\circ}\right)$. TES intensities that effectively phase-locked the spikes (see below) induced $2-3 \mathrm{mV}$ of intracellular polarization (Fig. 1D).
In the intact brain, neurons distant from the stimulation sites can be entrained directly through ephaptic coupling (Jefferys, 1995) or indirectly, through multisynaptic projections of the directly entrained neurons proximal to the stimulation sites. Support for the direct effect of TES comes from experiments in which recordings were made from multiple brain areas. In both acute and chronic experiments, recordings were made from both neocortex and hippocampus. As shown in Figure 2, $A$ and $B$, TESinduced entrainment in hippocampal and neocortical neurons was comparable ( $20 \%$ vs $16 \%$, respectively). In contrast, during slow oscillations of natural sleep, almost all neocortical neurons but only half of the CA1/subicular cells were phase-locked to the UP state of the slow oscillation (Fig. 2C). Importantly, the entrained neurons in the hippocampus/subiculum were phaselocked to the later phases of the slow oscillation than neocortical cells (Isomura et al., 2006). In contrast, spikes of both neocortical 

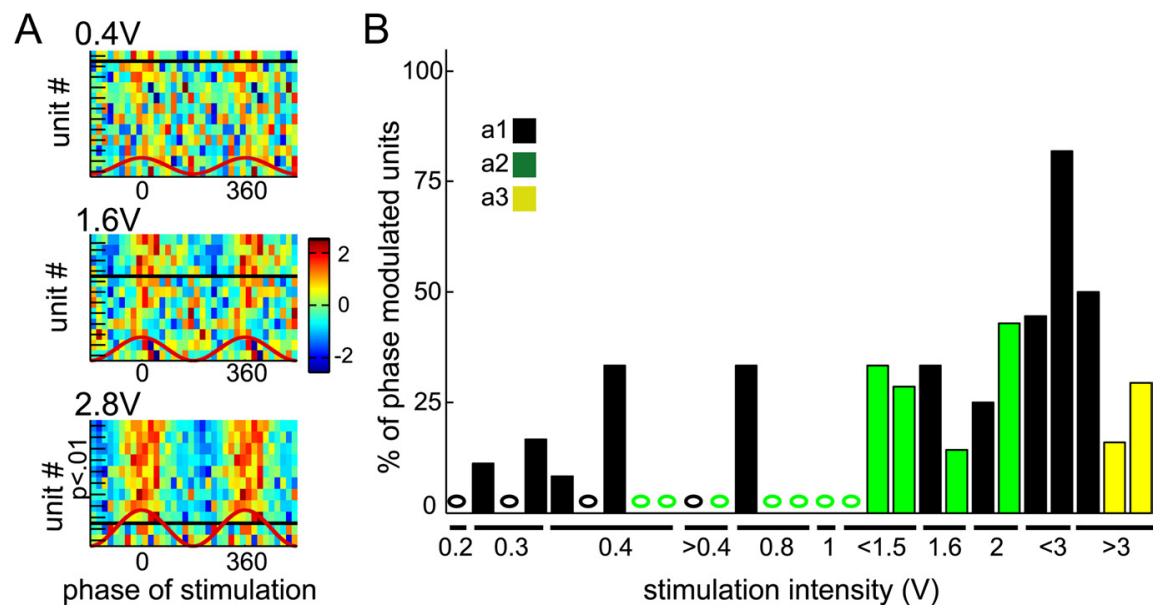

Figure 4. TES intensity-dependent phase-modulation of cortical unit activity. $A$, Illustration of TES intensity dependence of unit entrainment. Each row represents color-coded, normalized discharge probability of single-unit or multiunit firing as a function of the phase of TES at 3 different intensities $(0.4 \mathrm{~V}, 1.6 \mathrm{~V}, 2.8 \mathrm{~V})$ in animal a1. Units are ordered by the significance of their phase entrainment ( $p$-value, Kuiper's test); the unit with the smallest $p$-value is at the top. Black lines separate the significantly modulated units $(p<0.01)$ from the nonmodulated ones. $\boldsymbol{B}$. Percentage of significantly modulated neurons in 3 chronically implanted rats (a1, a2, a3) as a function of stimulation intensity. Each column represents a single recording session ( $1.25 \mathrm{~Hz}$, 3-pole configuration, as in Fig. $1 \mathrm{Aa}$ ). The lowest intensity tested was $0.2 \mathrm{~V}$. Intensities lower than $3 \mathrm{~V}$ were not tested in animal a3. The fraction of TES-entrained neurons is overestimated because neurons firing at a low rate are not detected properly by clustering algorithms.

and hippocampal/subicular neurons were locked to similar phases of TES. These findings support the hypothesis that at least a fraction of hippocampal neurons were directly affected by TES rather than indirectly influenced by TES-triggered neocortical neurons through multisynaptic actions. In a fraction of significantly modulated units, two prominent peaks at different phases coexisted, especially at higher stimulus intensities (Fig. 1C; supplemental Fig. 2, available at www.jneurosci.org as supplemental material). This finding might also be explained by both direct and indirect effect of TES on neurons. TES-induced phasemodulation was not specific to cell type, since both putative principal cells and interneurons were similarly modulated by TES ( $n=12$ putative excitatory and $n=11$ putative inhibitory cells were identified in $n=10$ chronic recording sessions and $n=17$ putative excitatory and $n=6$ putative inhibitory cells in $n=5$ acute sessions; supplemental Fig. 4, available at www.jneurosci.org as supplemental material).

Repeated long trains of TES in a given session generated stable neuronal entrainment over time (Fig. 3). Moreover, the spike phase-biasing effect of the TES appeared (disappeared) immediately after the onset (offset) of the stimulus (Fig. $3 B$ ). This observation is in support of the direct effect of TES on neuronal activity and also shows that current-induced polarization of the stimulation electrodes was negligible.

Previous experiments in vitro found a linear relationship between the amplitude of the applied-field and the induced transmembrane potential, suggesting the absence of a clear threshold (Deans et al., 2007). In accordance with these in vitro data, we found that weaker stimulation affected only a few neurons, while increasing stimulus intensity recruited an increasingly larger fraction of the population. Using $>1.2 \mathrm{~V}$ TES stimulation, a considerable fraction $(>20 \%)$ of the recorded units (single units and multiunits) showed significant phase-entrainment by the stimulus (Fig. $4 A, B$ ). Lower intensity stimulation elicited varying levels of entrainment and preferred phases (Figs. $1 C, 4 A, B$; supplemental Fig. $1 A, B$, available at www.jneurosci.org as supplemental material). With strong TES, the effects became much more ho- mogenous and a larger portion of the recorded neurons were significantly phasebiased by the forced field ( $p<0.05$; oneway ANOVA).

To estimate the magnitude of the TESinduced volume-conducted field at the recording sites, we first determined the approximate threshold current needed to entrain at least a few of the recorded neurons (Fig. 5D). Following the recording session, brain activity was abolished by a lethal dose of the anesthetic and the peakto-peak amplitude of the TES-induced potential was measured at multiple locations in the anteroposterior (Fig. $5 A, B$ ) or mediolateral (Fig. 5E) directions. As expected, the amplitude of the intracortical potential varied as a function of distance from the stimulation electrodes. The magnitude of the recorded local voltage varied threefold $(0.4-1.4 \mathrm{mV})$ in the anteroposterior direction but remained relatively constant across different cortical layers, indicating that the induced fields near the midline of the brain were comparable in the hippocampus and the overlying neocortex when epidural grids with relatively large surface area $(2.5 \times 4 \mathrm{~mm})$ were used as stimulating electrodes (Fig. $5 B)$. To estimate the minimum voltage gradient necessary to bias the spiking activity of single neurons, voltage measurements were also made in the mediolateral direction, i.e., parallel to the TESinduced current flow ( $8 \%$ significant modulation, $n=13$ units, Fig. 5E). These postmortem measurements indicate that $\sim 1$ $\mathrm{mV} / \mathrm{mm}$ in the extracellular space is sufficient to affect the discharge probability of cortical neurons. In our experiment, such effective voltage gradients were achieved by using $>1.2 \mathrm{~V}$ stimulation.

When multiple forces are at work, such as external TES effects and synaptically mediated network influences, they may either cooperate or compete against each other. We examined these interactions by applying the same intensity of TES in both naturally sleeping and actively exploring rat. As Figure 6, $A$ and $B$, illustrates, these behavioral states were defined by characteristically different LFP patterns: slow oscillation during sleep (interspersed with REM epochs) and $\sim 8 \mathrm{~Hz}$ theta oscillation of hippocampal origin in the ambulating rat (Steriade et al., 1993; Sirota and Buzsáki, 2005). At the intensities tested, none of the neurons were affected by TES stimulation $(1.25 \mathrm{~Hz})$ in the awake animal, while at least a fraction of the neurons (25-50\%) were significantly entrained during sleep (Fig. 6C). This finding suggests that TES, at least partially, entrained the slow oscillation of sleep. The lower TES intensity required to discharge neurons during sleep likely resulted from a combination of TES-induced fields and synaptically mediated effects.

\section{Discussion}

The main finding of the present experiments is that relatively weak electric fields applied through the skull can affect neurons in widespread cortical areas. The induced effects are brought about by an interaction between TES-induced field and ongoing network activities of the intact brain.

Electrical stimulation of neuronal elements is one of the oldest research tools in neuroscience (Fritsch and Hitzig, 1870). Cur- 
A

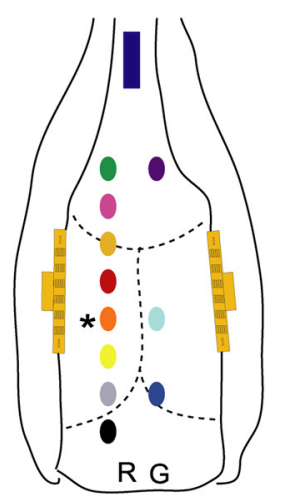

B

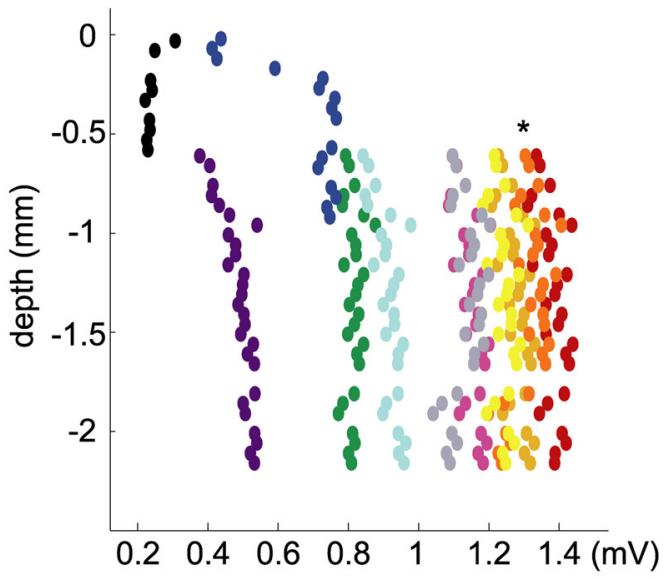

C

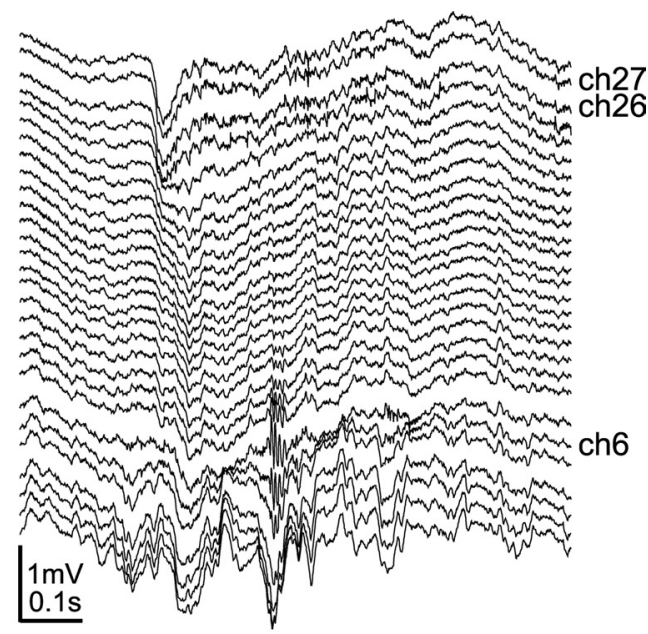

D

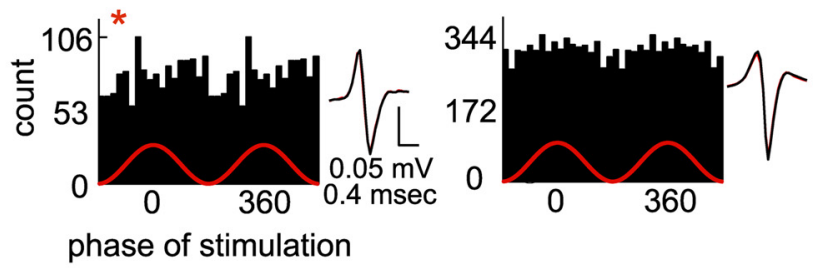

$\mathrm{E}$

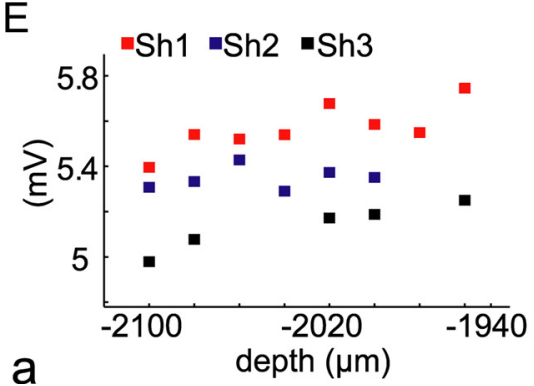

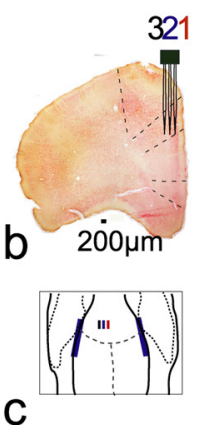

Figure 5. Determination of the magnitude of the TES-induced field. $A$, Locations of the penetration sites for measuring the volume-conducted local potentials $(0.8 \mathrm{~Hz} ; 3-$ pole configuration as in Fig. $1 \mathrm{Ad}$ ). Star indicates the anatomical location of unit recording. $\boldsymbol{B}$, Locally measured voltage between each active recording site, indicated by the color code in $A$, and the reference electrode, $R$. $\mathrm{G}$, ground. Depth measurements were performed with a 32 -site single-shank probe, with recording sites spaced at $50 \mu \mathrm{m}$ intervals along the vertical axis of the probe. TES, $0.8 \mathrm{~Hz}, 0.12 \mathrm{~mA}$ intensity. C, Short epoch of simultaneously recorded LFP from deep layers of the neocortex and the underlying hippocampus; orange site in $A$. Recording sites ch26 and ch27 correspond to layer V, while ch6 recorded from the CA1 pyramidal layer. Note ripple-related reversal of sharp wave in the CA1 str. radiatum. D, Example unit histograms during TES stimulation. Red star indicates the significant modulation ( $p<0.01$, Kuiper's test). Insets, averaged filtered unit waveforms recorded during prestimulation epochs (black) and during TES (red, superimposed). Measurements in B were performed after the rat was injected with a lethal dose of anesthetic, using the same intensity TES, which entrained the neurons. $\boldsymbol{E}$, Estimation of effective voltage gradient. $\boldsymbol{E b}$, $\boldsymbol{E} \boldsymbol{c}$, Three-shank probe placed parallel to the main current flow induced by TES electrodes. Ea, Postmortem voltage measurements at all probe sites. TES, $1.25 \mathrm{~Hz}, 1.2 \mathrm{~V}$ intensity. Note $\sim 400 \mu \mathrm{V}$ difference between shank 1 and shank $3(400 \mu \mathrm{m})$ at all depths.

rent applied by a small sized electrode can activate axon bundles, a local population of neurons or, under special conditions, a single cell, directly. Depending on the strength of the focal stimulation, neurons in more distant parts of the brain can be activated antidromically through their axons or anterogradely by mono- or multisynaptic connections (Histed et al., 2009). The goal of focal electrical stimulation under most conditions is to drive specific pathways or to identify neuronal groups with specific projections (Tepper and Groves, 1990). However, under many experimental and clinical conditions it is more desirable to affect large numbers of neurons distributed over large areas in a temporally precise manner without the need to identify the exact neuronal populations affected by the stimulation. Transcutaneous or transcranial magnetic or electric stimulation (TMS, TES) can meet these requirements. Early studies in anesthetized animals have shown that direct electric fields may affect the discharge frequency and amplitude of evoked potentials (Ranck, 1975; Jefferys, 1981). The magnitude of the polarization mostly depends on the geometry and the alignment of the neuron relative to the induced electric field, and also on the active channel properties of the cell (Chan and Nicholson, 1986; Chan et al., 1988; Lopez et al., 1991; Deans et al., 2007; Radman et al., 2007b, 2009). In addition to the single-cell features, cell density, extracellular resistance and other properties of the nervous tissue can modify the magnitude of the polarization experienced by neurons and glia (Jefferys, 1995; Francis et al., 2003). Importantly, networks in vivo may amplify the effect of stimulation (Saunders and Jefferys, 2007) because in the vicinity of the threshold, externally applied additional currents may either facilitate or prevent the discharge of neurons, depending on the polarity of the stimulation (Radman et al., 2007b).

In our experiments, both under anesthesia and in the behaving animal, at least a fraction of neurons recorded at distant sites from the stimulating electrodes could be affected by electric stimulation. The fraction of responding neurons as well as the magnitude of the induced effects was enhanced with increasing stimulation intensity. Under in vitro conditions, spatially homogeneous or "quasi-uniform" fields can be created by large stimulation plates (Jefferys and Haas, 1982; Chan and Nicholson, 1986; Chan et al., 1988; Bikson et al, 2004; Deans et al., 2007; Radman et al., 2007b), whereas in vivo the spread of the current from the limited size electrodes through the bone, meninges, CSF, vessels and intervening brain tissue may be quite complex. Our postmortem experiments indicate that $\sim 1 \mathrm{mV} / \mathrm{mm}$ TES-induced voltage gradient was sufficient to significantly affect neuronal excitability in at least a fraction of the neurons. This magnitude is comparable to that measured in previous in vitro experiments (Jefferys and Haas, 1982; Taylor and Dudek, 1982; Chan et al., 1988; 
A

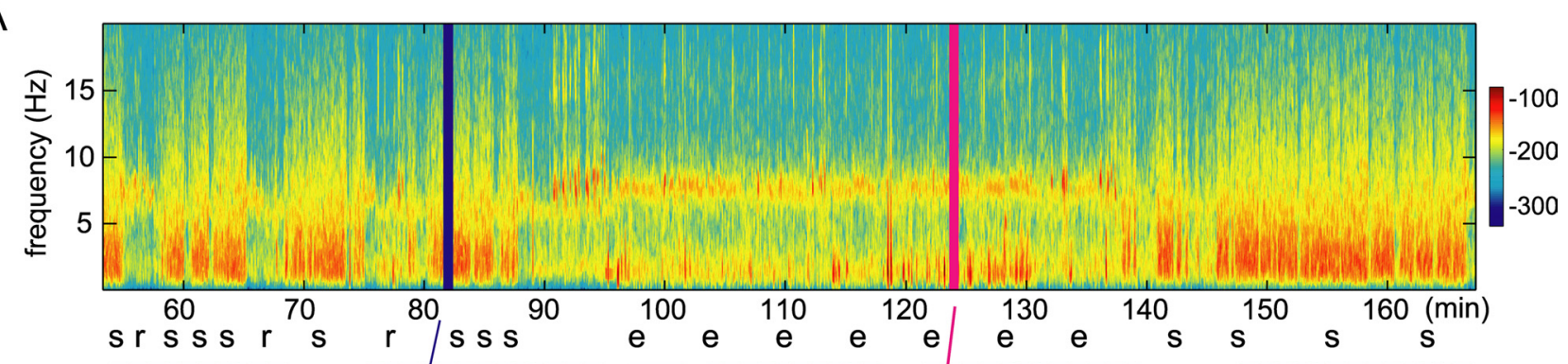

TES
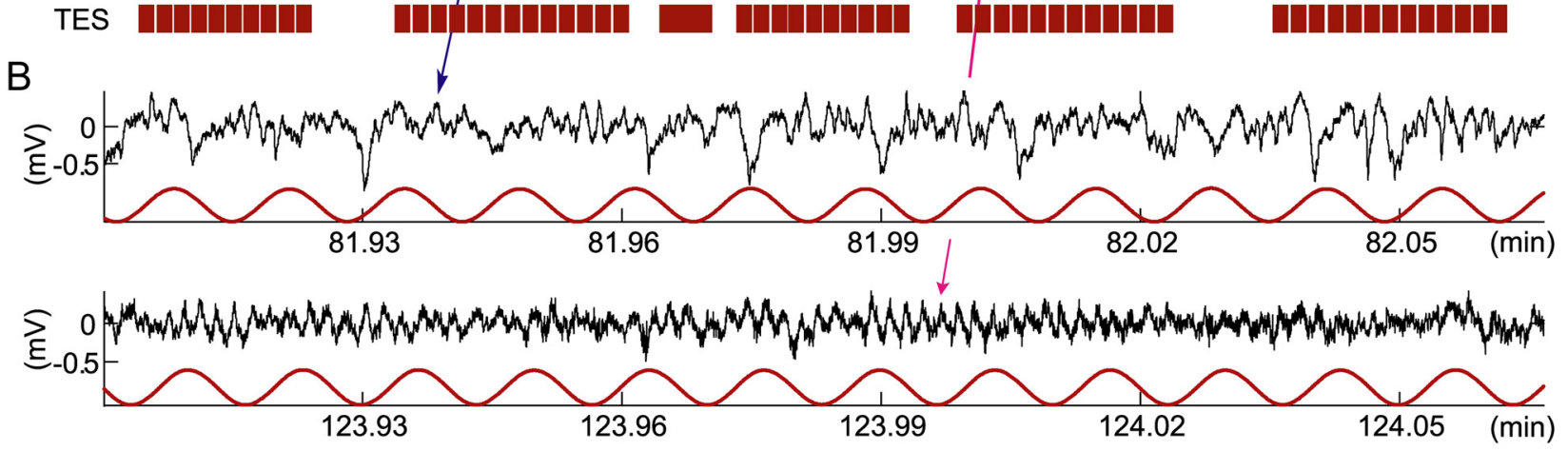

C
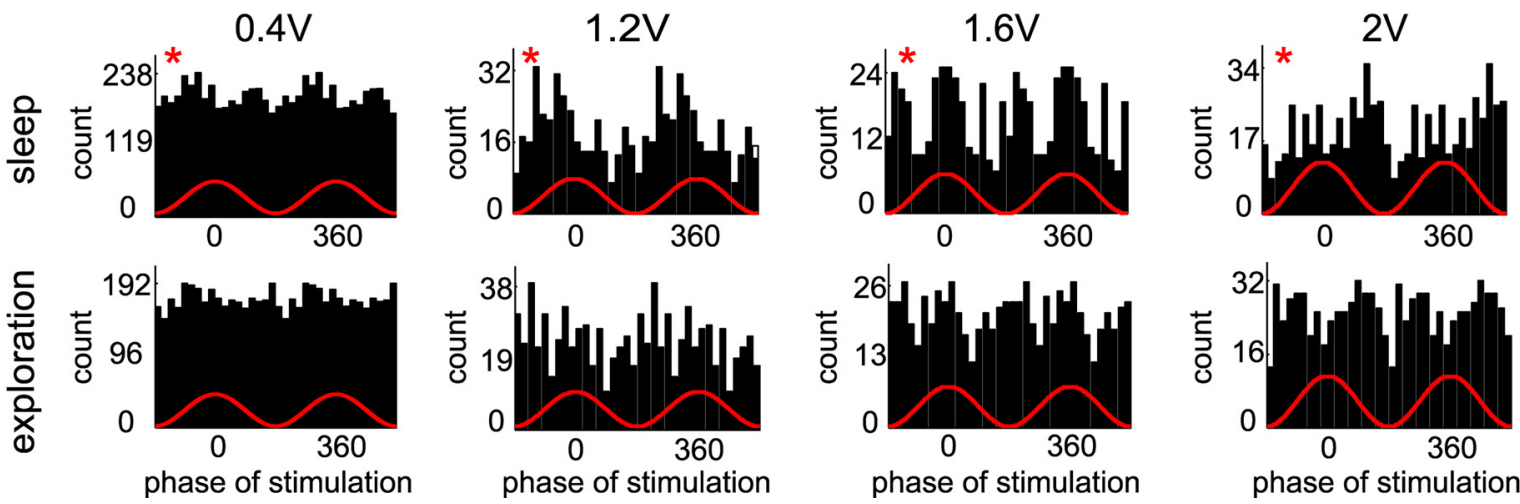

Figure 6. Network state dependence of TES entrainment. $A$, Spectrogram of power in band-passed filtered (1-500 Hz) signal from the CA1 pyramidal layer (from the tip of one of the shanks of 4-shank silicon probe, lowered to $\sim 2050 \mu \mathrm{m}$ from cortical surface). Sleep-related [alternation between slow-wave sleep (SWS) and REM epochs] and exploration-associated activity with dominant $8 \mathrm{~Hz}$ theta oscillation are distinguishable. Letters indicate state of vigilance defined by manual scoring. s, SWS; r, REM; e, exploration. The red boxes under the spectrum indicate stimulation trials. The color bar indicates the power in each frequency band of the time spectrum (arbitrary units). $\boldsymbol{B}$, Zoomed epochs taken from $A$ (blue and magenta lines) illustrate state-dependent LFP activity. TES, $1.25 \mathrm{~Hz}, 1.2 \mathrm{~V}$ intensity, 3-pole configuration as in Figure $1 \mathrm{Ad}$. C, Example unit histograms during TES. The electrodes were moved between session 1 ( $0.4 \mathrm{~V}$ ) and session 2 (1.2 V, 1.6 V, $2 \mathrm{~V}$ ); therefore, different sets of neurons were recorded in the two sessions but neuron identity in the sleep and exploration states is the same. Note that while no unit was affected by TES during exploration, a fraction of cells were significantly entrained by TES during sleep at all intensities.

Francis et al., 2003; Fujisawa et al., 2004; Deans et al., 2007; Radman et al., 2007b), to the estimated forced fields in human studies (Marshall et al., 2006; Kirov et al., 2009) and to the values predicted by modeling studies (Anastassiou et al., 2010).

Given the nonhomogeneous fields induced by our electrode configurations, it is expected that in the high-current-density neighborhood of the stimulating electrodes many neurons were affected directly and these neurons in turn could have polysynaptically recruited neurons in distant brain regions. In addition to such polysynaptic effects, our observations indicate that a sizable fraction of the significantly phase-locked neurons were directly driven by the TES forced field. First, approximately the same fraction of the recorded neocortical and hippocampal neurons were affected, and they were affected at similar phases of the sinusoid TES. In contrast, during intrinsic slow oscillations a relatively smaller fraction of hippocampal cells were affected and at a different phase of the slow oscillation compared with neocortical neurons (Isomura et al., 2006), suggesting the direct effect of
TES on, at least some of the, hippocampal neurons. Second, several neurons discharged at two different phases of TES forced field, especially at higher intensities. This may have occurred because the alternation of the virtual cathodal field differentially affected differently oriented processes, such as apical and basal dendrites of the same neuron, as demonstrated directly by previous in vitro experiments (Ranck, 1975; Radman et al., 2009) or because the same unit was driven both directly and polysynaptically. These findings indicate that in addition to polysynaptically evoked effects, TES can directly affect neurons in a large volume of the cortex even with the relatively small size electrodes and low stimulus intensities as used in our study.

Experimental and therapeutic implications of chronic TES Since cortical neurons are embedded in perpetually active networks, TES inevitably interacts with network-induced effects (Wiesenfeld and Moss, 1995; Saunders and Jefferys, 2007). Such interactions can take the form of amplification or interference. In 
the in vitro brain slice preparation, silent neurons have to be depolarized by several tens of millivolts to reach spiking threshold (but see Francis et al., 2003; Deans et al., 2007). In contrast, in the intact brain, large numbers of neurons are typically close to threshold in virtually any time window so that even very weak fields can have a time-biasing effect. TES in phase with networkinduced patterns can enhance neuronal discharge activity, as demonstrated here by the significantly stronger effect of the periodic TES during slow oscillation of sleep compared with the effects in the waking animal. Through the mechanism of stochastic resonance (Wiesenfeld and Moss, 1995; Gluckman et al., 1996b), even applied noise stimulation can exert a thresholdlowering effect and assist neuronal discharges. The noisy electric field, which in itself may be subthreshold, can be effectively summed with otherwise subthreshold effects of network-induced membrane voltage fluctuations and the combined effect can generate spikes in a fraction of the neuronal population. Such an amplification mechanism can have important consequences for both environmental safety issues (Adey, 1981; Bawin et al., 1984; Gluckman et al., 1996b; Jefferys et al., 2003) and for therapeutic stimulation technologies.

The effects of TES on spontaneous or evoked network patterns, demonstrated here, may be used for experimental manipulation of brain activity. Both regular (i.e., rhythmic) and irregular population network patterns are robustly expressed by the readily recordable local field potentials (Buzsáki and Draguhn, 2004). In turn, these patterns can be used to time the enhancing or suppressing effects of TES on spontaneous or sensory stimulation-evoked neuronal activity. Exploiting the temporal windows of spike timing-dependent plasticity (Levy and Steward, 1983; Magee and Johnston, 1997; Markram et al., 1997; $\mathrm{Bi}$ and Poo, 1998), such pairing may be used to increase or decrease the gain between synaptic inputs and spiking output noninvasively in the intact cortex (King et al., 1999). Similarly, abnormal patterns, such as epileptic spikes or other pathological rhythms can be used to trigger TES at various delays with the goal of attenuating or abolishing them (Gluckman et al., 1996a, 2001; Ghai et al., 2000; Francis et al., 2003).

Electrical fields can be applied by percutaneous electrodes (Marshall et al., 2006; Kanai et al., 2008) in acute experiments or by electrodes implanted subcutaneously or into the skull for chronic applications. In both cases, electrical fields can affect spiking activity of cortical neurons without intracortical tissue reaction, a typical problem of electrodes placed in the brain parenchyma (Polikov et al., 2005; van Kuyck et al., 2007). Importantly, nearly all effects of TMS on brain activity can be reproduced by applying electric fields via percutaneous or subcutaneous electrodes on the scalp. Electrodes implanted into the skull and powered by ultralight, minimal electrical circuits can eliminate stimulation of pain receptors in the skin or the periosteum. In contrast to the lissencephalic cortex of rodents, the convolution of the human cortex and consequently the systematic shifts of dendritic orientation in various gyri should be considered in clinical studies. On the other hand, the large brain size in humans allows for more restricted stimulation of specific brain areas by proper design of current delivery. The subtle but significant and widespread change in cortical excitability may be exploited for therapeutic effects in psychiatry.

Although the physiological effects of TES and TMS are comparable (Brocke et al., 2005), TMS requires large coils and complex instrumentation for powering the coils. In contrast, TES requires simple electronics, even when combined with EEG monitoring and waveform detection circuits; and the small device can be cosmetically hidden for long-term use in patients. Further experiments in chronically implanted animals are needed to further explore and evaluate the benefits and safety of TES.

\section{References}

Adey WR (1981) Tissue interactions with nonionizing electromagnetic fields. Physiol Rev 61:435-514.

Anastassiou CA, Montgomery SM, Barahona M, Buzsáki G, Koch C (2010) The effect of spatially inhomogeneous extracellular fields on neurons. J Neurosci 30:1925-1936.

Barker AT, Jalinous R, Freeston IL (1985) Non-invasive magnetic stimulation of human motor cortex. Lancet 1:1106-1107.

Barthó P, Hirase H, Monconduit L, Zugaro M, Harris KD, Buzsáki G (2004) Characterization of neocortical principal cells and interneurons by network interactions and extracellular features. J Neurophysiol 92:600-608.

Bawin SM, Sheppard AR, Mahoney MD, Adey WR (1984) Influences of sinusoidal electric fields on excitability in the rat hippocampal slice. Brain Res 323:227-237.

Bédard C, Kröger H, Destexhe A (2004) Modeling extracellular field potentials and the frequency-filtering properties of extracellular space. Biophys J 86:1829-1842.

Bi GQ, Poo MM (1998) Synaptic modifications in cultured hippocampal neurons: dependence on spike timing, synaptic strength, and postsynaptic cell type. J Neurosci 18:10464-10472.

Bikson M, Inoue M, Akiyama H, Deans JK, Fox JE, Miyakawa H, Jefferys JG (2004) Effects of uniform extracellular DC electric fields on excitability in rat hippocampal slices in vitro. J Physiol 557:175-190.

Bishop G, O'leary J (1950) The effects of polarizing currents on cell potentials and their significance in the interpretation of central nervous system activity. Electroencephalogr Clin Neurophysiol 2:401-416.

Brocke J, Irlbacher K, Hauptmann B, Voss M, Brandt SA (2005) Transcranial magnetic and electrical stimulation compared: does TES activate intracortical neuronal circuits? Clin Neurophysiol 116:2748-2756.

Buzsáki G, Draguhn A (2004) Neuronal oscillations in cortical networks. Science 304:1926-1929.

Chan CY, Nicholson C (1986) Modulation by applied electric fields of Purkinje and stellate cell activity in the isolated turtle cerebellum. J Physiol 371:89-114.

Chan CY, Hounsgaard J, Nicholson C (1988) Effects of electric fields on transmembrane potential and excitability of turtle cerebellar Purkinje cells in vitro. J Physiol 402:751-771.

Deans JK, Powell AD, Jefferys JG (2007) Sensitivity of coherent oscillations in rat hippocampus to AC electric fields. J Physiol 583:555-565.

Fisher NI (1993) Analysis of a single sample. In: Statistical analysis of circular data. Cambridge, UK: University of Cambridge.

Fitzgerald PB, Fountain S, Daskalakis ZJ (2006) A comprehensive review of the effects of rTMS on motor cortical excitability and inhibition. Clin Neurophysiol 117:2584-2596.

Francis JT, Gluckman BJ, Schiff SJ (2003) Sensitivity of neurons to weak electric fields. J Neurosci 23:7255-7261.

Fregni F, Boggio PS, Valle AC, Rocha RR, Duarte J, Ferreira MJ, Wagner T, Fecteau S, Rigonatti SP, Riberto M, Freedman SD, Pascual-Leone A (2006) A sham-controlled trial of a 5-day course of repetitive transcranial magnetic stimulation of the unaffected hemisphere in stroke patients. Stroke 37:2115-2122.

Fritsch G, Hitzig E (1870) Uber die elektrische Erregbarkeit des Grosshirns. Arch Anat Physiol Wissen 37: 300-332. [Reprinted in 2009: Electric excitability of the cerebrum. Epilepsy Behav 15:123-130.]

Fujisawa S, Matsuki N, Ikegaya Y (2004) Chronometric readout from a memory trace: gamma-frequency field stimulation recruits timed recurrent activity in the rat CA3 network. J Physiol 561:123-131.

Fujisawa S, Amarasingham A, Harrison MT, Buzsáki G (2008) Behaviordependent short-term assembly dynamics in the medial prefrontal cortex. Nat Neurosci 11:823-833.

George MS, Nahas Z, Kozel FA, Goldman J, Molloy M, Oliver N (1999) Improvement of depression following transcranial magnetic stimulation. Curr Psychiatry Rep 1:114-124.

Ghai RS, Bikson M, Durand DM (2000) Effects of applied electric fields on low-calcium epileptiform activity in the CA1 region of rat hippocampal slices. J Neurophysiol 84:274-280.

Gluckman BJ, Neel EJ, Netoff TI, Ditto WL, Spano ML, Schiff SJ (1996a) 
Electric field suppression of epileptiform activity in hippocampal slices. J Neurophysiol 76:4202-4205.

Gluckman BJ, Netoff TI, Neel EJ, Ditto WL, Spano ML, Schiff SJ (1996b) Stochastic resonance in a neuronal network from mammalian brain. Phys Rev Lett 77:4098-4101.

Gluckman BJ, Nguyen H, Weinstein SL, Schiff SJ (2001) Adaptive electric field control of epileptic seizures. J Neurosci 21:590-600.

Gold C, Henze DA, Koch C, Buzsáki G (2006) On the origin of the extracellular action potential waveform: a modeling study. J Neurophysiol 95:3113-3128.

Hallett M (2000) Transcranial magnetic stimulation and the human brain. Nature 406:147-150.

Harris KD, Henze DA, Csicsvari J, Hirase H, Buzsáki G (2000) Accuracy of tetrode spike separation as determined by simultaneous intracellular and extracellular measurements. J Neurophysiol 84:401-414.

Hazan L, Zugaro M, Buzsáki G (2006) Klusters, NeuroScope, NDManager: a free software suite for neurophysiological data processing and visualization. J Neurosci Methods 155:207-216.

Henze DA, Borhegyi Z, Csicsvari J, Mamiya A, Harris KD, Buzsáki G (2000) Intracellular features predicted by extracellular recordings in the hippocampus in vivo. J Neurophysiol 84:390-400.

Histed MH, Bonin V, Reid RC (2009) Direct activation of sparse, distributed populations of cortical neurons by electrical microstimulation. Neuron 63:508-522.

Holt GR, Koch C (1999) Electrical interactions via the extracellular potential near cell bodies. J Comput Neurosci 6:169-184.

Isomura Y, Sirota A, Ozen S, Montgomery S, Mizuseki K, Henze DA, Buzsáki G (2006) Integration and segregation of activity in entorhinal-hippocampal subregions by neocortical slow oscillations. Neuron 52:871-882.

Jefferys JG (1981) Influence of electric fields on the excitability of granule cells in guinea-pig hippocampal slices. J Physiol 319:143-152.

Jefferys JG (1995) Nonsynaptic modulation of neuronal activity in the brain: electric currents and extracellular ions. Physiol Rev 75:689-723.

Jefferys JG, Haas HL (1982) Synchronized bursting of CA1 hippocampal pyramidal cells in the absence of synaptic transmission. Nature 300: $448-450$.

Jefferys JG, Deans J, Bikson M, Fox J (2003) Effects of weak electric fields on the activity of neurons and neuronal networks. Radiat Prot Dosimetry 106:321-323.

Kanai R, Chaieb L, Antal A, Walsh V, Paulus W (2008) Frequencydependent electrical stimulation of the visual cortex. Curr Biol 18: 1839-1843.

King C, Henze DA, Leinekugel X, Buzsáki G (1999) Hebbian modification of a hippocampal population pattern in the rat. J Physiol 521:159-167.

Kirov R, Weiss C, Siebner HR, Born J, Marshall L (2009) Slow oscillation electrical brain stimulation during waking promotes EEG theta activity and memory encoding. Proc Natl Acad Sci U S A 106:15460-15465.

Koch C (1999) Biophysics of computation: information processing in single neurons. New York: Oxford UP.

Levy WB, Steward O (1983) Temporal contiguity requirements for longterm associative potentiation/depression in the hippocampus. Neuroscience 8:791-797.

Logothetis NK, Kayser C, Oeltermann A (2007) In vivo measurement of cortical impedance spectrum in monkeys: implications for signal propagation. Neuron 55:809-823.

Lopez L, Chan CY, Okada YC, Nicholson C (1991) Multimodal characterization of population responses evoked by applied electric field in vitro: extracellular potential, magnetic evoked field, transmembrane potential, and current-source density analysis. J Neurosci 11:1998-2010.

Magee JC, Johnston D (1997) A synaptically controlled, associative signal for Hebbian plasticity in hippocampal neurons. Science 275:209-213.

Markram H, Lübke J, Frotscher M, Sakmann B (1997) Regulation of synaptic efficacy by coincidence of postsynaptic APs and EPSPs. Science 275:213-215.

Marshall L, Helgadóttir H, Mölle M, Born J (2006) Boosting slow oscillations during sleep potentiates memory. Nature 444:610-613.

McIntyre CC, Grill WM (1999) Excitation of central nervous system neurons by nonuniform electric fields. Biophys J 76:878-888.

Nitsche MA, Boggio PS, Fregni F, Pascual-Leone A (2009) Treatment of depression with transcranial direct current stimulation (tDCS): a review. Exp Neurol 219:14-19.
Noebels JL, Prince DA (1978) Excitability changes in thalamocortical relay neurons during synchronous discharges in cat neocortex. J Neurophysiol 41:1282-1296.

Olsson RH 3rd, Buhl DL, Sirota AM, Buzsaki G, Wise KD (2005) Bandtunable and multiplexed integrated circuits for simultaneous recording and stimulation with microelectrode arrays. IEEE Trans Biomed Eng 52:1303-1311.

Parra LC, Bikson M (2004) Model of the effect of extracellular fields on spike time coherence. Conf Proc IEEE Eng Med Biol Soc 6:4584-4587.

Polikov VS, Tresco PA, Reichert WM (2005) Response of brain tissue to chronically implanted neural electrodes. J Neurosci Methods 148:1-18.

Radman T, Datta A, Peterchev AV (2007a) In vitro modulation of endogenous rhythms by AC electric fields: Syncing with clinical brain stimulation. J Physiol 584:369-370.

Radman T, Su Y, An JH, Parra LC, Bikson M (2007b) Spike timing amplifies the effect of electric fields on neurons: implications for endogenous field effects. J Neurosci 27:3030-3036.

Radman T, Ramos RL, Brumberg JC, Bikson M (2009) Role of cortical cell type and morphology in subthreshold and suprathreshold uniform electric field stimulation in vitro. Brain Stimulation 2:215-228.

Rall W, Shepherd GM (1968) Theoretical reconstruction of field potentials and dendrodendritic synaptic interactions in olfactory bulb. J Neurophysiol 31:884-915.

Ranck JB Jr (1975) Which elements are excited in electrical stimulation of mammalian central nervous system: a review. Brain Res 98:417-440.

Rosenthal SH (1972) Electrosleep therapy. Curr Psychiatr Ther 12:104-107.

Sanchez-Vives MV, McCormick DA (2000) Cellular and network mechanisms of rhythmic recurrent activity in neocortex. Nat Neurosci 3:1027-1034.

Saunders RD, Jefferys JG (2007) A neurobiological basis for ELF guidelines. Health Phys 92:596-603.

Sirota A, Buzsáki G (2005) Interaction between neocortical and hippocampal networks via slow oscillations. Thalamus Relat Syst 3:245-259.

Sirota A, Csicsvari J, Buhl D, Buzsáki G (2003) Communication between neocortex and hippocampus during sleep in rodents. Proc Natl Acad Sci U S A 100:2065-2069.

Sirota A, Montgomery S, Fujisawa S, Isomura Y, Zugaro M, Buzsáki G (2008) Entrainment of neocortical neurons and gamma oscillations by the hippocampal theta rhythm. Neuron 60:683-697.

Steriade M, Nuñez A, Amzica F (1993) A novel slow ( $<1 \mathrm{~Hz}$ ) oscillation of neocortical neurons in vivo: depolarizing and hyperpolarizing components. J Neurosci 13:3252-3265.

Taylor CP, Dudek FE (1982) Synchronous neural afterdischarges in rat hippocampal slices without active chemical synapses. Science 218:810-812.

Tepper JM, Groves PM (1990) In vivo electrophysiology of central nervous system terminal autoreceptors. Ann N Y Acad Sci 604:470-487.

Terney D, Chaieb L, Moliadze V, Antal A, Paulus W (2008) Increasing human brain excitability by transcranial high frequency random noise stimulation. J Neurosci 28:14147-14155.

Terzuolo CA, Bullock TH (1956) Measurement of imposed voltage gradient adequate to modulate neuronal firing. Proc Natl Acad Sci U S A 42:687694.

Tranchina D, Nicholson C (1986) A model for the polarization of neurons by extrinsically applied electric fields. Biophys J 50:1139-1156.

Traub RD, Dudek FE, Taylor CP, Knowles WD (1985) Simulation of hippocampal afterdischarges synchronized by electrical interactions. Neuroscience 14:1033-1038.

Traub RD, Jefferys JG, Whittington MA (1999) Functionally relevant and functionally disruptive (epileptic) synchronized oscillations in brain slices. Adv Neurol 79:709-724.

van Kuyck K, Welkenhuysen M, Arckens L, Sciot F, Nuttin B (2007) Histological alterations induced by electrode implantation and electrical stimulation in the human brain: a review. Neuromodulation 10:244-261.

Walsh V, Cowey A (2000) Transcranial magnetic stimulation and cognitive neuroscience. Nat Rev Neurosci 1:73-79.

Walsh V, Pascual-Leone A (2003) Transcranial magnetic stimulation: a neurochronometrics of mind. Cambridge, MA: MIT.

Webster BR, Celnik PA, Cohen LG (2006) Noninvasive brain stimulation in stroke rehabilitation. NeuroRx 3:474-481.

Wiesenfeld K, Moss F (1995) Stochastic resonance and the benefits of noise: from ice ages to crayfish and squids. Nature 373:33-36. 